\title{
Use of Hounsfield units of S1 body to diagnose osteoporosis in patients with lumbar degenerative diseases
}

\author{
Da Zou, MD, ${ }^{1,2}$ Weishi Li, MD, ${ }^{1}$ Fei Xu, BM, ${ }^{1}$ and Guohong Du${ }^{1}$ \\ ${ }^{1}$ Orthopaedic Department, Peking University Third Hospital; and ${ }^{2}$ Peking University Health Science Center, Haidian District, \\ Beijing, People's Republic of China
}

\begin{abstract}
OBJECTIVE The aim of this study was to evaluate the use of Hounsfield unit (HU) values of the S1 body to diagnose osteoporosis in patients with lumbar degenerative diseases.

METHODS The records of 316 patients of ages $\geq 50$ years and requiring surgery for lumbar degenerative diseases were reviewed. The bone mineral density (BMD) of the $\mathrm{S} 1$ body and $\mathrm{L} 1$ was measured in $\mathrm{HU}$ with preoperative lumbar CT. Circular regions of interest (ROIs) were placed on midaxial and midsagittal images of the S1 body. Dual-energy $x$-ray absorptiometry (DXA) and the criterion of $\mathrm{L} 1 \mathrm{HU} \leq 110 \mathrm{HU}$ were used to diagnose osteoporosis. The area under the receiver operating characteristic curve (AUC) was calculated to assess the use of HUs of the S1 body to diagnose osteoporosis.
\end{abstract}

RESULTS The interobserver and intraobserver reliability of measuring $\mathrm{HU}$ of the $\mathrm{S} 1$ body was excellent with intraclass correlation coefficients over $0.8(p<0.001)$. The correlation between HUs of the S1 body and average T-score of L1-4 was significant with Pearson correlation coefficients $\geq 0.60$ ( $p<0.001)$. The AUCs for using HUs of the $S 1$ body to diagnose osteoporosis were 0.86 and 0.88 for axial $\mathrm{HU}$ and sagittal $\mathrm{HU}$, respectively $(\mathrm{p}<0.001)$. The HU thresholds with balanced sensitivity and specificity for diagnosing osteoporosis were $202 \mathrm{HU}$ for axial HU (sensitivity: 76\%; specificity: $76 \%$ ) and $185 \mathrm{HU}$ for sagittal HU (sensitivity: $80 \%$; specificity: $80 \%$ ).

CONCLUSIONS Both sagittal and axial HUs of the S1 body are useful tools for assessing BMD and diagnosing osteoporosis. Measuring HUs of the S1 body preoperatively from lumbar CT may help with surgical planning for patients with lumbar degenerative diseases.

https://thejns.org/doi/abs/10.3171/2019.2.FOCUS18614

KEYWORDS sacrum; osteoporosis; Hounsfield units; bone mineral density; lumbar degenerative disease

$\mathrm{T}$ HE first sacral vertebra (S1) is always chosen as the caudal level for spinal fusion with instrumentation for lumbar degenerative diseases because the L5-S1 level has the highest prevalence of disc degeneration in the lumbar spine, which may require decompression and fusion. ${ }^{15}$ In addition, long fusion to S1 for lumbar deformity is associated with better maintenance of sagittal alignment and a lower rate of subsequent disc degeneration of L5-S1. ${ }^{3}$ However, fusion to S1 can also have a high rate of complications such as pedicle screw loosening or pseudarthrosis, especially for older patients with osteoporosis. ${ }^{7,19}$

An S1 body with poor bone stock can cause screw loosening in the bone-implant interface, leading to the failure of fixation. ${ }^{18,19}$ Therefore, preoperative measurement of the bone mineral density (BMD) of the S1 body is essential for surgical planning, which helps surgeons decide whether or not to use other techniques to prevent failure at S1.,18 Dual-energy x-ray absorptiometry (DXA) is a widely used method to evaluate BMD and diagnose osteoporosis, but it is routinely used to measure BMD of the first to fourth lumbar vertebrae (L1-4) and hips. ${ }^{4}$ Based on the positive correlation between the BMD of different sites in the same patient, the BMD of the S1 body can usually only be estimated via DXA rather than measured directly. ${ }^{19}$ Moreover, DXA can overestimate the BMD of the lumbar spine and cause a missed diagnosis of osteoporosis in lumbar degenerative disease, especially for degenerative lumbar scoliosis, which is a more common disease needing fusion to $\mathrm{S} 1 .^{2,10,11}$

Since 2011, the method of using Hounsfield units (HUs)

ABBREVIATIONS AUC = area under the curve; BMD = bone mineral density; DXA = dual-energy $x$-ray absorptiometry; HU = Hounsfield unit; ICC = intraclass correlation coefficient; $\mathrm{ROI}=$ region of interest.

SUBMITTED November 5, 2018. ACCEPTED February 18, 2019.

INCLUDE WHEN CITING DOI: 10.3171/2019.2.FOCUS18614. 
from clinical CT scanning has been widely recommended to assess BMD and screen for osteoporosis. ${ }^{1,8,13,14,17} \mathrm{HU}$ values of lumbar vertebrae from L1 to L5 have excellent reliability, significant correlation with DXA T-scores, and good performance in diagnosing osteoporosis. Observers are able to reduce the effect of lumbar degenerative changes on $\mathrm{HU}$ values by manually choosing appropriate regions of interest (ROIs) for measurement. Since lumbar CT is a common examination performed for patients requiring surgery for lumbar degenerative diseases, HUs can be used to evaluate BMD at no extra cost and radiation. There have been a few studies involving the manual measurement of HUs of the S1 body from clinical CT scans, ${ }^{5,6,16}$ which support the use of HUs to determine sacral BMD. However, the correlation of HUs of the S1 body with DXA T-scores and the diagnosis of osteoporosis has not been explored. The present study aimed to evaluate the use of HUs of the S1 body to diagnose osteoporosis and to establish HU thresholds if possible.

\section{Methods \\ Patients}

We reviewed the records of consecutive patients requiring lumbar surgery for lumbar degenerative diseases at our spine center for the period between July 1, 2015, and December 31, 2015. Inclusion criteria were 1) an age of 50 years or older and 2) lumbar CT scanning and central DXA scanning within a month before surgery. Exclusion criteria were 1) a history of spinal surgery; 2) the presence of spinal tuberculosis, tumor, ankylosing spondylitis, diffuse idiopathic skeletal hyperostosis, and other diseases that could significantly affect bone metabolism and BMD; and 3 ) the presence of severe degenerative changes in the intervertebral disc or endplate at L5-S1 with no appropriate place for measuring HUs of the trabecular bone in the S1 body.

\section{BMD Evaluation}

Preoperative lumbar 3D reconstructive CT (tube voltage $120 \mathrm{kV}$, DEFINITION, Siemens) and picture archiving and communication system (PACS) were used to calculate HU values. Patients with transitional vertebrae were not excluded. The L1 vertebral level was identified as the first non-rib-bearing vertebra, and the level of S1 was counted from L1. HU values of the L1 body were measured according to the method of Pickhardt et al. ${ }^{13}$ As for S1, a circular ROI was placed over the midaxial and midsagittal images of the S1 body, respectively (Fig. 1). The diameter of the axial ROI was half of the anteroposterior diameter of the $\mathrm{S} 1$ body on the midaxial image. Because of the relatively small sagittal image of the S1 body, the rule in placing the sagittal ROI was including as much trabecular bone as possible and avoiding cortical bone and heterogeneous areas. All of the HU values mentioned above were measured by the first author (D. Z.). A month later, the first author measured the HU values of the S1 body of 30 patients picked randomly to evaluate intraobserver reliability. The second author (W.L.) also measured the HU values of the S1 body of these 30 patients to evaluate interobserver reliability. When measuring the HU values, the two observers
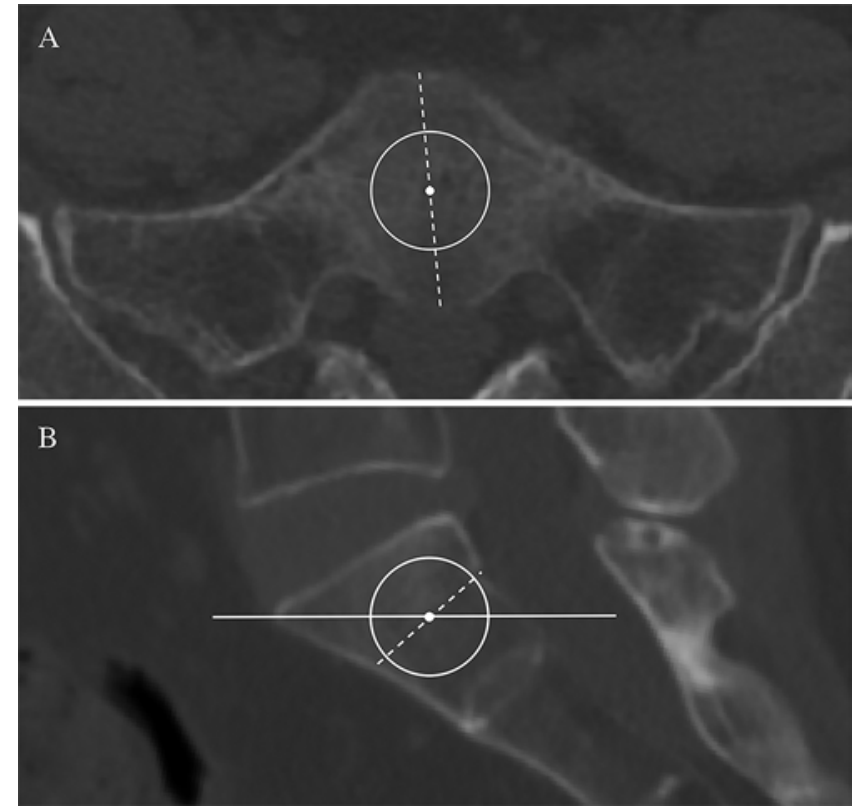

FIG. 1. The method of measuring HUs of the S1 body. Image (A) derived from the axial plane at the level of the horizontal white line in panel $\mathbf{B}$. The axial ROI was placed in the center of the axial image, and its diameter was half of the anteroposterior diameter of the S1 body. The sagittal $\mathrm{ROI}$ was placed in the center of the midsagittal image in $\mathrm{B}$. The rule in placing the sagittal ROI was including as much trabecular bone as possible and avoiding cortical bone and heterogeneous areas.

were blinded to the T-scores and the measurements of the other observer.

Central (L1-4, femoral neck, and total hip) DXA (Discover A densitometers, Hologic Inc.) was used to diagnose osteoporosis according to the WHO criterion: T-score $\leq$ 2.5 at any skeletal site. Considering that DXA can overestimate the BMD of the lumbar spine in patients with lumbar degenerative diseases, the criterion of $\mathrm{L} 1 \mathrm{HU} \leq 110 \mathrm{HU}$ in a previous study was also used to reduce missed diagnoses of osteoporosis. ${ }^{13}$ Therefore, patients were diagnosed with osteoporosis if they had a T-score $\leq-2.5$ or L1 $\mathrm{HU} \leq 110$ HU.

\section{Statistical Analysis}

Statistical analysis was conducted using SPSS software (version 20, IBM Corp.). The intraclass correlation coefficient (ICC) was used to evaluate interobserver and intraobserver reliability (ICC $\geq 0.8$ was considered to indicate excellent reliability). The correlation between $\mathrm{HU}$ values and T-scores was calculated with the Pearson correlation coefficient. The paired t-test was used for comparing the HU values of different vertebral bodies. Receiver operating characteristic curve (ROC) analysis and area under the curve (AUC) were used to evaluate the values of $\mathrm{HU}$ in diagnosing osteoporosis. HU thresholds for diagnosing osteoporosis were adjusted to the nearest integer for the ease of clinical use.

\section{Results}

A total of 316 patients (143 males and 173 females) were included in this study, and the mean patient age was $61.5 \pm$ 
TABLE 1. Pearson correlation coefficients between measurements of BMD

\begin{tabular}{lccc}
\hline \multicolumn{1}{c}{ Parameter } & Axial HU & Sagittal HU & L1 HU \\
\hline Sagittal HU & $0.95^{*}$ & & \\
\hline $\mathrm{L} 1 \mathrm{HU}$ & $0.80^{*}$ & $0.80^{*}$ & \\
\hline Average T-score of L1-4 & $0.60^{*}$ & $0.61^{*}$ & $0.63^{*}$ \\
\hline${ }^{*} p<0.001$. & & &
\end{tabular}

6.9 years old (range 50-81 years). Interobserver reliability in measuring axial $\mathrm{HU}$ and sagittal $\mathrm{HU}$ was excellent with ICCs of 0.971 and 0.967 , respectively. Intraobserver reliability in measuring axial $\mathrm{HU}$ and sagittal $\mathrm{HU}$ was also excellent with ICCs of 0.990 and 0.983 , respectively. The correlations among axial HU, sagittal HU, L1 HU, and average T-score of L1-4 are shown in Table 1. The sagittal HU was lower than axial HU $(185.8 \pm 59.2 \mathrm{HU}$ vs 206.0 $\pm 60.5 \mathrm{HU}, \mathrm{p}<0.001)$. The L1 HU was lower than sagittal HU $(124.2 \pm 38.5 \mathrm{HU}$ vs $185.8 \pm 59.2 \mathrm{HU}, \mathrm{p}<0.001)$.

The prevalence of osteoporosis identified by DXA and L1 HU $\leq 110 \mathrm{HU}$ was $50.9 \%$ (161/316). The AUCs for using axial HU and sagittal HU to distinguish these osteoporotic patients from non-osteoporotic patients were $0.86(95 \% \mathrm{CI}$ $0.82-0.90$ ) and 0.88 (95\% CI 0.84-0.92), respectively (Fig. 2). The axial HU threshold of 202 HU had balanced sensitivity and specificity to diagnose osteoporosis (sensitivity: 76\%; specificity: 76\%). The axial HU thresholds with high sensitivity $(90 \%)$ or high specificity $(90 \%)$ were 222 HU and $183 \mathrm{HU}$, respectively. The sagittal HU threshold of $185 \mathrm{HU}$ had balanced sensitivity and specificity to diagnose osteoporosis (sensitivity: 80\%; specificity: $80 \%$ ). The sagittal HU thresholds with high sensitivity (90\%) or high specificity (90\%) were $200 \mathrm{HU}$ and $160 \mathrm{HU}$, respectively.

When we only used DXA to identify osteoporotic patients, the prevalence of osteoporosis was $40.5 \%$ (128/316), and the AUCs for using axial HU and sagittal HU to distinguish these osteoporotic patients were also over 0.8 (axial HU: 0.82, 95\% CI 0.77-0.87; sagittal HU: 0.83, 95\% CI 0.78-0.87).

\section{Discussion}

Results of this study showed that both axial HU and sagittal HU of the S1 body had positive correlations with DXA T-scores and good performance in diagnosing osteoporosis in patients with lumbar degenerative diseases. The axial HU threshold of $202 \mathrm{HU}$ and sagittal HU threshold of $185 \mathrm{HU}$ had balanced sensitivity and specificity over $75 \%$ for distinguishing osteoporosis from non-osteoporosis. Two examples are given to show how to use these HU thresholds to detect osteoporosis (Fig. 3).

Similar to the methods used in previous studies, $, 5,6,16$ the ROIs in our study were manually placed in the center of midaxial and midsagittal images of the S1 bodies. The reliability of our measurements was excellent with ICCs comparable to those in the study by Katsuura et al. ${ }^{6}$ To our knowledge, this is the first study to show the significant correlation between HUs of the S1 body and DXA T-scores, and we found that their Pearson correlation coefficients were comparable to those between HUs of L1-5

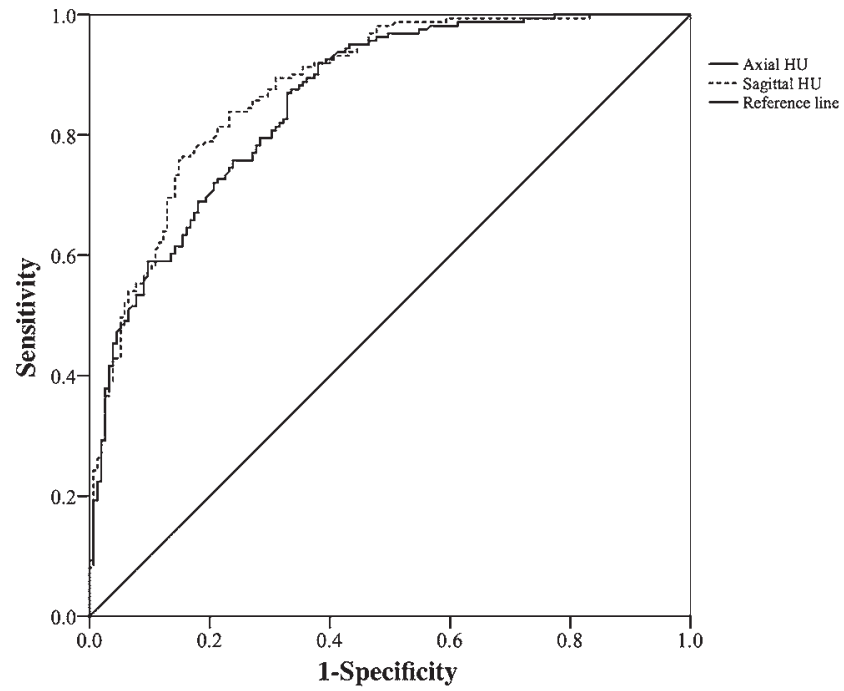

FIG. 2. Receiver operating characteristic curves for distinguishing osteoporosis identified by DXA or L1 HU $\leq 110 \mathrm{HU}$ from non-osteoporosis with axial $\mathrm{HU}$ and sagittal $\mathrm{HU}$, respectively.

and DXA T-scores in other studies. ${ }^{8,17}$ However, these coefficients of $0.6-0.7$ were relatively small, which may be the result of choosing patients with older ages and lumbar degenerative diseases. According to Choi et al., ${ }^{2}$ the Pearson correlation coefficients could increase to 0.7 if they had only analyzed the data of patients with mild lumbar degeneration.

There was a significant difference among HUs of different sites: L1 HU < sagittal HU of S1 body < axial HU of S1 body. Hoel et al. ${ }^{5}$ also found that HU of L1 (165 HU) was lower than HU of S1 body (220 HU). This implies that we need different thresholds for osteoporosis to assess
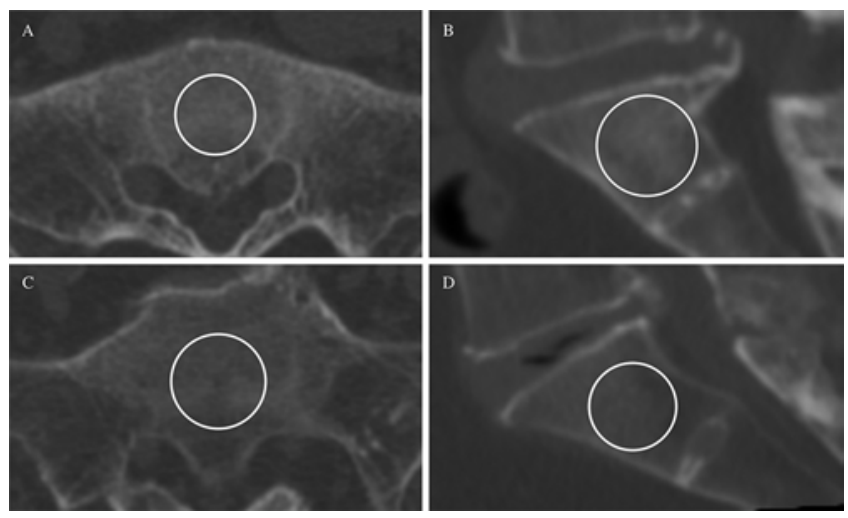

FIG. 3. Clinical examples of using HU thresholds of the $S 1$ body to detect osteoporosis. Images (A and B) from a 55-year-old female with normal BMD; T-scores of 0.5 at $L 1,-0.3$ at $L 2,0.5$ at $L 3,-0.2$ at $L 4,-1$ at the femoral neck, and 0.2 at the total hip; and an L1 at $197 \mathrm{HU}$. Her axial $\mathrm{HU}(\mathrm{A})$ and sagittal HU (B) were 311.7 and 284.7, respectively. According to our thresholds with balanced sensitivity and specificity, she was diagnosed with non-osteoporosis. Images ( $\mathbf{C}$ and $\mathbf{D}$ ) from a 67-year-old female with osteoporosis; T-scores of -2.1 at $L 1,-3.2$ at $L 2,-1.1$ at $L 3$, -1.6 at $L 4,-2.7$ at the femoral neck, and -2.1 at total hip; and an $L 1$ at $78.3 \mathrm{HU}$. Her axial HU (C) and sagittal HU (D) were 170.3 and 121.2, respectively. According to our thresholds with balanced sensitivity and specificity, she was diagnosed with osteoporosis. 
the BMD of different sites. For example, an average HU value of $175 \mathrm{HU}$ for L1-5 is highly suggestive of normal $\mathrm{BMD},{ }^{8}$ but an $\mathrm{HU}$ of $175 \mathrm{HU}$ for the S1 body may suggest osteoporosis.

Because of the effect of lumbar degenerative changes on DXA measurements, the diagnosis determined by DXA may not be accurate. Thus, we used the threshold of L1 $\mathrm{HU}$ to reduce missed diagnoses of osteoporosis as much as possible. The level of L1 has the lowest rate of degeneration when compared with lower levels (L2-S1) and was recommended for measuring HUs because it can be easily identified on different kinds of CT scans. ${ }^{13,15}$ The results showed that the prevalence of osteoporosis and the AUCs were higher when we combined the criterion of DXA and L1 HU $\leq 110$ HU instead of using the criterion of DXA alone. In both ways, we found that the use of axial HU and sagittal HU of the S1 body to diagnose osteoporosis was excellent with AUCs over 0.8, which were also comparable to the AUCs (0.8-0.9) of using HU of L1-L5 to diagnose osteoporosis. ${ }^{1,8,13}$ To provide more information about $\mathrm{HU}$ of the $\mathrm{S} 1$ body for clinical use, we identified the HU thresholds of different sensitivities and specificities. An HU threshold of balanced sensitivity and specificity may give general information about the correlation between $\mathrm{HU}$ and the diagnosis of osteoporosis. An HU threshold of high sensitivity can identify patients who need further evaluation of BMD. Furthermore, patients meeting the HU threshold of high specificity are very likely to have an osteoporotic S1 body despite the coexistence of relatively normal T-scores or HU values for L1-5. In line with the order of HU values of different sites (L1 HU < sagittal HU of S1 body < axial HU of S1 body), the thresholds with the same level of sensitivity or specificity were also highest at the axial S1 body and lowest at L1. For example, the threshold of $90 \%$ specificity was $183 \mathrm{HU}$ at the axial S1 body, $160 \mathrm{HU}$ at the sagittal HU body, and $90-110 \mathrm{HU}$ at L1. ${ }^{12,13}$

Usually, we can only estimate BMD of the S1 body with the T-scores of L1-4, whereas our study provides a simple method of directly evaluating it. The HUs of the S1 body and corresponding thresholds for osteoporosis can give us more information about the bone stock of a specific $\mathrm{S} 1$ body before lumbar surgery. Moreover, lumbar CT is commonly performed preoperatively for patients requiring lumbar fusion; the measurement of $\mathrm{HU}$ demands no further investment in equipment and personnel training. Patients having high $\mathrm{HU}$ values can avoid extra radiation from DXA, and those having relatively lower HU values according to our thresholds can avoid being misdiagnosed with non-osteoporosis because of lumbar degeneration.

There are several limitations to our study. Firstly, it is a retrospective single-center study focusing on patients ages 50 years or older with lumbar degenerative diseases. Thus, we still need to be careful when we plan to apply the findings to other populations. Secondly, manual measurement of HUs lacks certain quality assurance protocols used in DXA and quantitative CT (QCT). ${ }^{12}$ Therefore, although the measurement of $\mathrm{HU}$ is economic and simple, it should be used as a complementary method to DXA rather than a replacement. Thirdly, the prevalence of disc degeneration at L5-S1 is higher than that at other levels, and the S1 body is relatively small compared with L1-5 vertebral bodies.
As a result, the degenerative changes around or at the upper endplate of S1 might make it difficult to find appropriate ROIs of trabecular bone for measuring HUs, in which case the patients were excluded from this study.

\section{Conclusions}

Both axial and sagittal HUs of the S1 body are reliable tools with good performance in diagnosing osteoporosis in patients with lumbar degenerative diseases. Measuring HUs of the S1 body preoperatively from lumbar CT may help with surgical planning for patients with lumbar degenerative diseases.

\section{References}

1. Alacreu E, Moratal D, Arana E: Opportunistic screening for osteoporosis by routine CT in Southern Europe. Osteoporos Int 28:983-990, 2017

2. Choi MK, Kim SM, Lim JK: Diagnostic efficacy of Hounsfield units in spine CT for the assessment of real bone mineral density of degenerative spine: correlation study between T-scores determined by DEXA scan and Hounsfield units from CT. Acta Neurochir (Wien) 158:1421-1427, 2016

3. Edwards CC II, Bridwell KH, Patel A, Rinella AS, Berra A, Lenke LG: Long adult deformity fusions to L5 and the sacrum. A matched cohort analysis. Spine (Phila Pa 1976) 29:1996-2005, 2004

4. Hamdy RC, Petak SM, Lenchik L: Which central dual X-ray absorptiometry skeletal sites and regions of interest should be used to determine the diagnosis of osteoporosis? J Clin Densitom 5 (Suppl):S11-S18, 2002

5. Hoel RJ, Ledonio CGT, Takahashi T, Polly DW Jr: Sacral bone mineral density (BMD) assessment using opportunistic CT scans. J Orthop Res 35:160-166, 2017

6. Katsuura Y, Lorenz E, Gardner W II: Anatomic parameters of the sacral lamina for osteosynthesis in transverse sacral fractures. Surg Radiol Anat 40:521-528, 2018

7. Kim YJ, Bridwell KH, Lenke LG, Rhim S, Cheh G: Pseudarthrosis in long adult spinal deformity instrumentation and fusion to the sacrum: prevalence and risk factor analysis of 144 cases. Spine (Phila Pa 1976) 31:2329-2336, 2006

8. Li YL, Wong KH, Law MWM, Fang BXH, Lau VWH, Vardhanabuti VV, et al: Opportunistic screening for osteoporosis in abdominal computed tomography for Chinese population. Arch Osteoporos 13:76, 2018

9. Luk KDK, Chen L, Lu WW: A stronger bicortical sacral pedicle screw fixation through the S1 endplate: an in vitro cyclic loading and pull-out force evaluation. Spine (Phila Pa 1976) 30:525-529, 2005

10. Muraki S, Yamamoto S, Ishibashi H, Horiuchi T, Hosoi T, Orimo $\mathrm{H}$, et al: Impact of degenerative spinal diseases on bone mineral density of the lumbar spine in elderly women. Osteoporos Int 15:724-728, 2004

11. Pappou IP, Girardi FP, Sandhu HS, Parvataneni HK, Cammisa FPJ Jr, Schneider R, et al: Discordantly high spinal bone mineral density values in patients with adult lumbar scoliosis. Spine (Phila Pa 1976) 31:1614-1620, 2006

12. Pickhardt PJ, Lauder T, Pooler BD, Muñoz Del Rio A, Rosas $\mathrm{H}$, Bruce RJ, et al: Effect of IV contrast on lumbar trabecular attenuation at routine abdominal CT: correlation with DXA and implications for opportunistic osteoporosis screening. Osteoporos Int 27:147-152, 2016

13. Pickhardt PJ, Pooler BD, Lauder T, del Rio AM, Bruce RJ, Binkley N: Opportunistic screening for osteoporosis using abdominal computed tomography scans obtained for other indications. Ann Intern Med 158:588-595, 2013 
14. Pompe E, de Jong PA, de Jong WU, Takx RAP, Eikendal ALM, Willemink MJ, et al: Inter-observer and inter-examination variability of manual vertebral bone attenuation measurements on computed tomography. Eur Radiol 26:30463053, 2016

15. Sabnis AB, Chamoli U, Diwan AD: Is L5-S1 motion segment different from the rest? A radiographic kinematic assessment of 72 patients with chronic low back pain. Eur Spine J 27:1127-1135, 2018

16. Schönenberg D, Guggenberger R, Frey D, Pape HC, Simmen HP, Osterhoff G: CT-based evaluation of volumetric bone density in fragility fractures of the pelvis - a matched casecontrol analysis. Osteoporos Int 29:459-465, 2018

17. Schreiber JJ, Anderson PA, Rosas HG, Buchholz AL, Au AG: Hounsfield units for assessing bone mineral density and strength: a tool for osteoporosis management. J Bone Joint Surg Am 93:1057-1063, 2011

18. Yu BS, Zhuang XM, Zheng ZM, Zhang JF, Li ZM, Lu WW: Biomechanical comparison of 4 fixation techniques of sacral pedicle screw in osteoporotic condition. J Spinal Disord Tech 23:404-409, 2010

19. Zhuang XM, Yu BS, Zheng ZM, Zhang JF, Lu WW: Effect of the degree of osteoporosis on the biomechanical anchoring strength of the sacral pedicle screws: an in vitro compari- son between unaugmented bicortical screws and polymethylmethacrylate augmented unicortical screws. Spine (Phila Pa 1976) 35:E925-E931, 2010

\section{Disclosures}

The authors report no conflict of interest concerning the materials or methods used in this study or the findings specified in this paper.

\section{Author Contributions}

Conception and design: Zou. Acquisition of data: Li, Zou. Analysis and interpretation of data: all authors. Drafting the article: Zou. Critically revising the article: all authors. Reviewed submitted version of manuscript: all authors. Approved the final version of the manuscript on behalf of all authors: Li. Statistical analysis: Zou, Xu. Study supervision: Li.

\section{Correspondence}

Weishi Li: Peking University Third Hospital, Beijing, China. wslee_puth@163.com. 